\title{
Integrated modernization of the gas-and-air system of a turbocharged diesel engine (21/21)
}

\author{
Leonid Plotnikov ${ }^{1, *}$, Nikolay Kochev ${ }^{1}$, and Leonid Osipov ${ }^{1}$ \\ ${ }^{1}$ Ural Federal University named after the first President of Russia B.N. Yeltsin, Yekaterinburg, Russia
}

\begin{abstract}
Improving the exploitative and environmental performance of piston engines (PICE) is an urgent task for many engineers and scientists. The article presents the results of the upgrade of a gas-and-air system of a diesel PICE, carried out through changing the turbocharging system's configuration and modernizing the design of the admittance collector. The authors present a review of studies on the given subject and a description of the object of the research. The study was conducted on the basis of bench tests at a manufacturing plant and mathematical modeling using ACTUS program. The results of experimental studies on the main indicators of a basic and upgraded PICEs are presented. The gas exchange processes in the PICE under examination were studied in detail using mathematical modeling. For the given diesel PICE, improvement of the gas-and-air system leads to a growth in charging efficiency by $2.45-3.92 \%$, a decrease in scavenging factor by $3.11-6.31 \%$ and a reduction of specific fuel consumption up to $3.33 \%$. In the conclusion, new directions for increasing the efficiency of the given PICE are offered.
\end{abstract}

\section{Introduction}

Today, piston internal combustion engines (PICE) are some of the most convenient and mobile sources of energy. They can operate on various types of fuel (gasoline, gas, biofuels, alcohol, hydrogen, etc.). Improving the performance, efficiency and environmental friendliness of ICE is the main goal in the development of this industry. Their performance depends not only on improving processes in the engine cylinder, but also on the quality of the cylinder filled with a working substance and the degree to which said cylinder is clean of end gases [1,2]. These processes determine the amount of the working medium at the start of combustion and its physical and chemical properties, thus having a direct influence on the exploitative indicators of power systems based on PICEs.

Many engineers and scientists have perfected the designs of the admittance and final systems of PICEs. It is possible to single out works where mathematical modeling is used to evaluate the influence of the spiral canal in the cylinder head on PICE's exploitative and ecological indicators [3-5]. It is demonstrated that the use of a spiral canal results in a growth in engine power by up to $6.05 \%$ and the reduction of NOx emission up to $40.55 \%$ compared to the basic system. Other experts have explored the possibility of developing an admittance manifold in order to grow the performance of PICEs through numerical simulation in different programs [6-8]. It has been stated that a refined configuration of admittance and final systems can grow the airflow rate in the system by $10.5-25.2 \%$, which also leads to increased PICE power [9-11]. Thus, the authors assess the influence of the GaAS's configuration on the processes taking place inside the cylinder. It is notable that studies using mathematical modeling to examine heat and mass transfer processesin the admittance and final systems have often been confirmed by the results of experiments $[12,13]$. Still, it is important to note that experimental studies are carried out under steady-state conditions. It is obvious that the PICE working processes are periodic with a high cyclicity. Vibrations, high temperatures, external turbulence, etc. should be considered in research as well. Modern PICE research should be carried out in conditions as close as possible to reality. The turbocharging system (TCS) is actively used in PICE in order to grow their exploitative performance [14]. Effective application of TCS in diesel PICE has revealed a number of problems and the tasks for further improvement of gas-and-air systems (GaAS). Modern requirements for the exploitative and ecological indices of PICEs with TCS are closely related to the strict requirements for gas-dynamics and heat exchange in GaAS [15-17]. Many studies are aimed at designing GaAS s with optimum geometrical forms and without sources for the possible appearance of stagnant zones [18-20]. Still, the maximum effect is achieved by the integrated upgrade of the admittance and final systems, TCS adjustment and engine development [21, 22]. Such upgrade results in a growth in PICE power by up to $28 \%$ and the reduction of specific fuel consumption (SFC) $b_{e}$ by $2.54 \%$ on average, compared to a basic PICE.

This work describes the results of bench tests and numerical modeling as applied to a universal diesel ICE of dimension 210/210 (cylinder diameter - $210 \mathrm{~mm}$, piston stroke - $210 \mathrm{~mm}$ ). During the study, a

* Corresponding author: leonplot@mail.ru 
comprehensive upgrade of the GaAS was carried out by changing the TCS and fine-tuning the admittance collector. It is important to obtain reliable information about these processes.

\section{Description of the study subject}

As a study subject, the authors chose a diesel PICE produced by the Ural Diesel-Motor Plant (Russian Federation, Yekaterinburg). It is an eight-cylinder 4stroke engine with a V-type cylinder arrangement at an angle of $90^{\circ}$, with direct fuel injection, a closed-type liquid cooling system, gas turbine charging, aftercooling, and a dry-sump lubrication system. The engine's power rating is $980 \mathrm{~kW}$ at a crankshaft speed of $1500 \mathrm{rpm}$ and a maximum torque of $1500 \mathrm{~N} \cdot \mathrm{m}$. The factory designation of the unit is 8UDMP-21. In order to define the directions for improving diesel engine 8UDMP-21, the authors have conducted bench tests at a manufacturing plant and mathematical modeling of working processes using ACTUS program, developed by ABB Turbo Systems. Let us take a brief look at the basic schematic of the GaAS in the PICE (Figure 1). Arrows indicate the direction of gas movement.

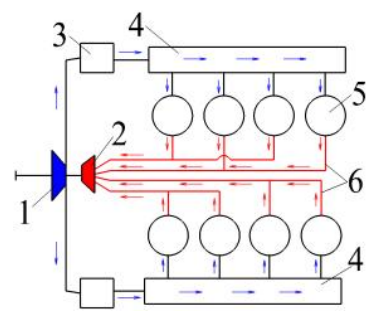

Fig. 1. Schematic of the gas-and-air system of 8UDMP-21: 1compressor; 2 - turbine; 3 - aftercooler; 4 - admittance collector; 5 - diesel cylinders; 6 - final collector.

In figure 1, the basic GaAS has one turbocharger produced by LLC "Special turbocharger design bureau" (Russia). Air, compressed in the centrifugal compressor, goes through the pipelines to the admittance collectors on the left and right sides of the V-type diesel. The turbine case has four inlets. In the given configuration, the final hoods from two cylinders form a single pipeline in order to reduce the pressure wave energy. It should be noted that the 8UDMP-21 has a traditional layout.

\section{Results of the studies and analysis}

As a result of studies on the examined diesel engine, the following basic characteristics were obtained (Figure 2). The minimum effective $b_{e}$ is about $212 \mathrm{~g} /(\mathrm{kW} \cdot \mathrm{h})$, and the maximum about $250 \mathrm{~g} /(\mathrm{kW} \cdot \mathrm{h})$. These values of $b_{e}$ are high: it is necessary to reduce them in order to grow the engine's competitiveness. Actual air consumption through the PICE is 0.182 to $0.802 \mathrm{~kg} / \mathrm{s}$ in the idle mode and nominal mode, respectively. Along with this, the compressor pressure ratio ranges from 1.08 to 2.38 , while the air ratio $\alpha$ ranges from 1.62 to 2.23. The minimum values of $\alpha$ for the initial diesel PICE are close to engine smoking, which was observed visually during the tests, which is undesirable under operational conditions. Obviously, upgrade of the TCS is necessary.

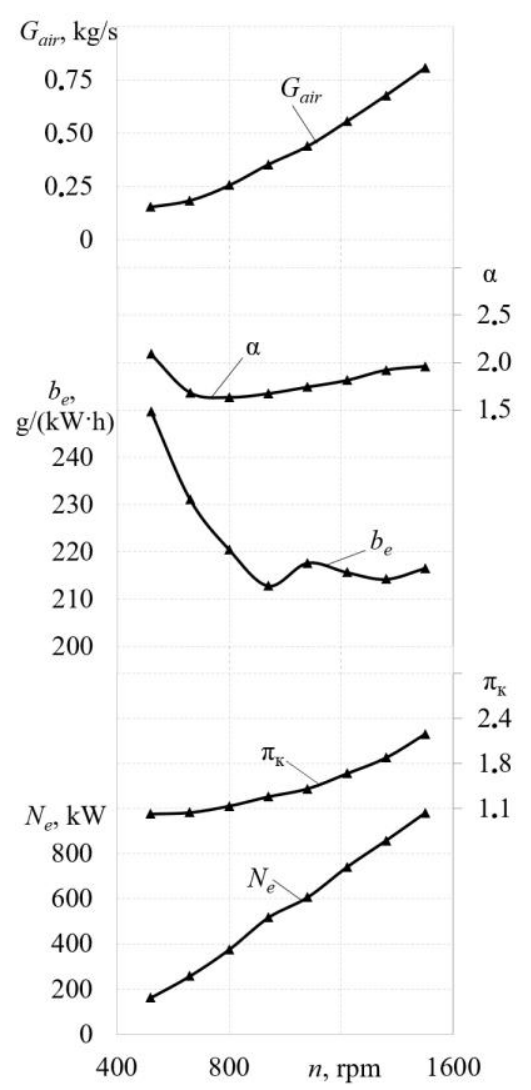

Fig. 2. Basic characteristics of the 8UDMP-21: $G_{a i r}$-actual air consumption through the cylinders; $\alpha$ - air ratio; $b_{e}$ - effective SFC; $\pi_{\mathrm{K}}$ - compressor pressure ratio; $N_{e}$ - effective power.

Modeling of the PICE working process was carried out in the ACTUS program. A mathematical model of the 8UDMP-21 was developed. It included the main systems of this PICE. The program has a block structure. Each block has its own physical properties and mathematical description. A set of interconnected blocks defines a particular system or engine element. The simulation results of the base PICE were correlated with the test results. Differences in the main indicators did not exceed $5.22 \%$. The program simulates the working processes of PICEs with a TCS with fairly high accuracy. This is confirmed by a large number of studies comparing the results of modeling and bench tests of PICEs. Some examples can be found in [22, 23].

The results of modeling the gas dynamics in the GaAS are presented in Figure 3. At the moment the admittance port is opened, the pressure in the final collector is higher than in the admittance one (this area is circled in Figure 3). This points to the fact that products get into the admittance collector in the process of inlet combustion, which, in turn, may lead to a deficiency of oxygen, necessary for complete fuel combustion, and, consequently, to decreased ecological indices and excessive smoke. Previously, this design was used to reduce the size of the PICE. 


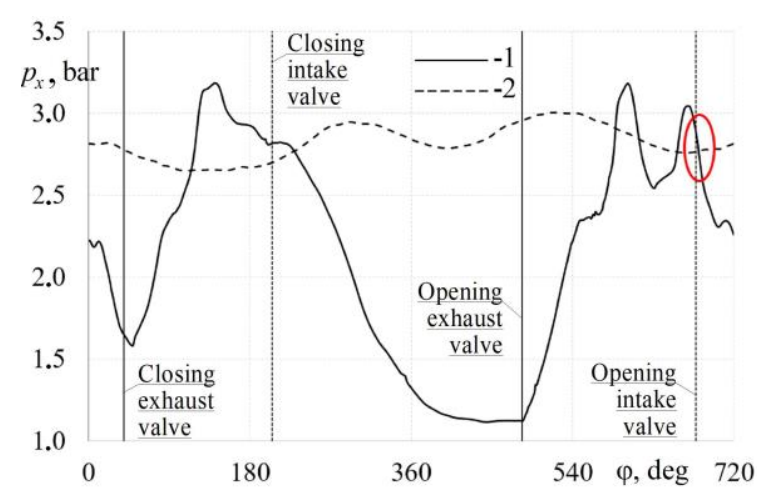

Fig. 3. Estimated dependence of pressures $p_{x}$ in the admittance port (1) and the final port (2) for the third left cylinder of the 8UDMP-21 on the crankshaft rotation angle $\varphi$ in the nominal operational mode.

The basic configuration of this engine's admittance collector has strongly marked technical deficiencies in terms of its gas-dynamics (Figure 4). There are fixture elements in the way of the air flow, which cause vortices and reduce the flow section (they are circled in Figure 4b). This can lead to deterioration in the quality of the cylinder filled with a working substance. This will lead to a reduction in charging efficiency, a growth in exhaust smoke, a growth in $b_{e}$ (reduction in the efficiency of the PICE and TCS).

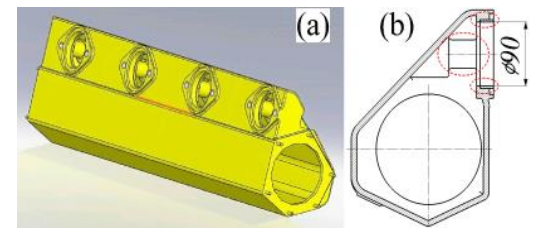

Fig. 4. General view of the admittance collector (a) and its cross section (b) for the basic 8UDMP-21.

The principal directions of gas-dynamic improvement for the GaAS of engine 8UDMP-21 were established, which consist of changing the TCS's configuration (in order to grow the air ratio $\alpha$ and refine carburation and combustion) and upgrading the construction of the admittance collector (with the aim of improving charging efficiency and reducing SFC $b_{e}$ ).

On the basis of literature analysis, the scheme of the TCS was changed. A pulse TCS with two small turbochargers produced by ABB Turbo Systems was used. This design solution allowed for the compressor pressure ratio to be increased, thus increasing the actual air consumption through the PICE. The TCS's response time was also reduced, which should grow PICE acceleration compared to a basic PICE equipped with one large turbocharger. Concurrently, the configuration of the admittance collectors was upgraded, namely, their in-line and cross-cut profiling was performed (Figure 5). The new design is more modern. The key changes are related to the change in the geometry of the admittance ports to each cylinder: this is aimed at minimizing the sources of flow swirling. The proposed design of the admittance collector has smoother surfaces compared to the base collector. At the same time, fasteners on the path of air movement are absent in the new design. It should be noted that the main characteristics of the bore sections were saved in this design. Thus, the proposed design has a reduced hydraulic resistance, and therefore, has refined flow characteristics. This should lead to a growth in charging efficiency and a reduction in fuel consumption for the 8UDMP-21.

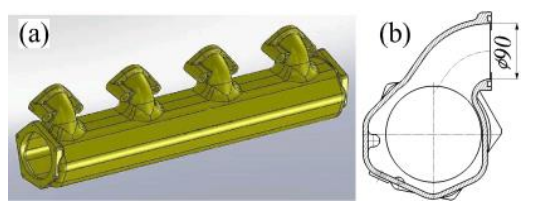

Fig. 5. General view of the upgraded admittance collector (a) and its cross-section (b) for 8UDMP-21.

After selecting a new TCS scheme and applying new admittance collectors, the upgraded 8UDMP-21 was tested (Figure 6).

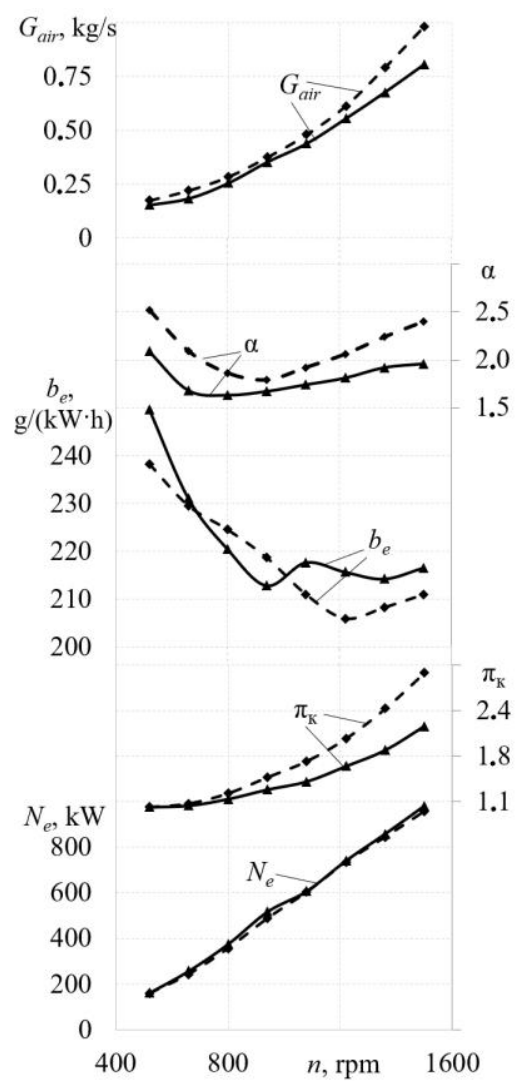

Fig. 6. Speed characteristics of the basic (1) and upgraded (2) 8UDMP-21: the designations in this figure are identical to Figure 2.

The figure shows the combined dependencies for both PICEs. In Figure 6 , the $N_{e}$ of the upgraded diesel PICE in partial modes is slightly higher (by 5-7\%) than that of a basic diesel. Presumably, this effect was achieved by increasing the compressor pressure ratio $\pi_{\mathrm{K}}$, and, consequently, the actual air consumption, which led to increased charging efficiency, along with refined carburation and combustion. In this case, the compressor pressure ratio ranges from 1.12 to 2.49 , while the air ratio $\alpha$ ranges from 1.81 to 2.52 . The values of the $\alpha$ coefficient are far from the values characteristic of the exhaust smoke. 
The charging efficiency of the upgraded diesel increased by $2.45-3.92 \%$. The growth of charging efficiency occurs in all operating modes of the PICE and TCS. The charging efficiency determines the degree to which the cylinder is filled with air. This should lead to an increase in the efficiency of both the PICE and the turbocharger. The smoke of the gases also decreased. Moreover, the upgrade of the GaAS also led to a reduction in scavenging factor by $3.11-6.31 \%$ compared with the base system. This indicates an improvement in exhaust cleaning of the cylinders as well. Reducing the scavenging factor will also increase the efficiency of the TCS, as the gas flow through the final system will grow. The charging efficiency and scavenging factor are key indicators of gas exchange and have a direct relationship with each other.

\section{Conclusions}

Application of the new configuration of the admittance collector and two turbochargers with refined exploitative and flow characteristics increased the compressor pressure ratio (by about $8.02 \%$ ) and the charging pressure (on average by $10.5-15.3 \%$ ). It also resulted in an increased air ratio (by $5.42 \%$ on average) in partial modes, which had a positive effect on the processes of carburation and combustion, leading to reduced smoke and emission toxicity. Despite the relatively small growth in the power of the upgraded PICE, the effective SFC $b_{e}$ was reduced by about $3.11 \%$ in some modes, which will allow for a reduction in the overall fuel consumption by the end user of up to $10.51 \mathrm{~kg} / \mathrm{h}$. Using a TCS with two small turbochargers also refined the mass and size characteristics of the PICE and increased its response. This grows the competitiveness of the 8UDMP-21.

As an additional potential direction for further upgrade of the 8UDMP-21, we can consider thermomechanical improvement of the final system (a thorough refinement of the final collectors) and studying the application of a constant-pressure TCS in front of the turbine. The directions of improving the GaAS of PICEs considered in the article are universal. The results of this study can be extended to PICEs of other dimensions in order to refine their exploitative indicators.

\section{Acknowledgements}

The work has been supported by the Russian Science Foundation (grant No. 18-79-10003).

\section{References}

[1] C.R. Ferguson, A.T. Kirkpatrick, Internal combustion engines: applied thermosciences (New York: John Wiley \& Sons, 2016)

[2] W.W. Pulkrabek, Engineering Fundamentals of the Internal Combustion Engine (New Jersey: Prentice Hall, Upper Saddle River, 2005)
[3] A.M. Sadeq, M.A. Bassiony, A.M. Elbashir, S.F. Ahmed, M. Khraisheh, Fuel 255, UNSP 115769 (2019)

[4] G.X. Wang, W.B. Yu, X.B. Li, Y.P. Su, R. Yang, W.T. Wu, Energy 180, 1008-1018 (2019)

[5] E.A.A. Silva, A.A.V. Ochoa, J.R. Henriquez, Energy conversion and management 188, 310320 (2019)

[6] S.Q. Guo, S. Huang, M.S. Chi, Eurasip J. image and video processing $\mathbf{7 0}$ (2018)

[7] G.K. Giannakopoulos, C.E. Frouzakis, K. Boulouchos, P.F. Fischer, A.G. Tomboulides, Int. J. heat and fluid flow 68, 257-268 (2017)

[8] S.D. Hanriot, J.M. Queiroz, C.B. Maia, J. Brazilian society of mechanical sciences and engineering 41, 2, UNSP 79 (2019)

[9] L. Kapitza, O. Imberdis, H.P. Bensler, J. Willand, D. Thevenin, Experiments in fluids 48, 2, 265280 (2010)

[10] G.J. Micklow, W.D. Gong, Proc. institution of mechanical engineers part d-J. automobile engineering 221, D11, 1425-1440 (2007)

[11] E. Yilmaz, M. Ichiyanagi, T. Suzuki, Int. J. automotive technology 20, 5, 1065-1071 (2019)

[12] D.R. Jebamani, T.M.N. Kumar, Thermal science 12, 1, 25-32 (2008)

[13] M.A. Ceviz, M. Akin, Energy conversion and managemen 51, 11, 2239-2244 (2010)

[14] N. Watson, M.S. Janota, Turbocharging the Internal Combustion Engine (London, Palgrave Macmillan Press, 1982)

[15] L.V. Plotnikov, IOP Conf. Series: J. Physics 899, 042008 (2017)

[16] L.V. Plotnikov, B.P. Zhilkin, Applied Thermal Engineering 160, 114123 (2019)

[17] M.W. Bae, Y.J. Ku, H.S. Park, Tran. Korean society of mechanical engineers B 43, 5, 379-388 (2019)

[18] U.B. Khairuddin, A.W. Costall, J. Engineering for Gas Turbines and Power 140, 5, 052801 (2018)

[19] Y.M. Brodov, L.V. Plotnikov, B.P. Zhilkin Technical Physics 63, 3, 319-324 (2018)

[20] G.X. Wang, W.B. Yu, X.B. Li, R. Yang, Fuel 262, UNSP 116548 (2020)

[21] M.A. Jemni, G. Kantchev, M.S. Abid, Energy 36, 5, 2701-2715 (2011)

[22] L.V. Plotnikov, S. Bernasconi, Y.M. Brodov, Procedia Engineering 206, 140-145 (2017)

[23] L.V. Plotnikov, S. Bernasconi, P. Jacoby, Lecture Notes in Mechanical Engineering, 549-558 (2020) 